\title{
A Novel Location Method for Multi-Target Based on the Maximum Likelihood Estimation
}

\author{
Xiantong $\mathrm{Ma}^{\mathrm{a}}$, Jingqing $\mathrm{Luo}^{\mathrm{b}}$ \\ Electronic Engineering Institute, Hefei, China \\ amxtzhy@foxmail.com, ${ }^{b}$ luojingqing001@163.com
}

\begin{abstract}
Keywords: maximum likelihood estimation; position information field; location; multi-target
Abstract. In the passive location system, the traditional maximum likelihood estimation (MLE) location method can't eliminate larger error or wrong parameter automatically, and does not apply to multi-target situation. A novel location method named as position information field for target (PIFT) location method is proposed for multi-target based on the MLE location method. By adding a cost function, the PIFT location method can overcome the disadvantage of MLE location method mentioned above and adapt to the complex electromagnetic environment. It is suitable for the multi-target localization and determines target's number and position simultaneously even the measured parameters of multi-target are indistinguishable. The effectiveness and adaptive of the proposed method is verified by computer experiments.
\end{abstract}

\section{Introduction}

In the traditional passive location system, the signals of radiation source are detected firstly by observation platform, and the measuring parameters related to the positioning of targets such as direction of arrival[1], time of arrival [2] and time difference of arrival [3,4] are also obtained by observation platform though some certain algorithm. We can use these measured parameters and the positioning of the station itself to estimate the positioning of radiation sources through grid search, iteration or analytic method and so on. Maximum likelihood estimation (MLE) location method [5] is a kind of typical method used in passive location system, which using all the parameters information obtained by the platform to estimate the positioning of radiation source. MLE location method has a good positioning performance for single objective when the errors of the measured parameters are small.

However, in the actual complex electromagnetic environment, measurement parameters errors are larger, and even get error parameters. Take the classic direction-finding method [6] as an example, we should use some estimation algorithm such as MUSIC [7], ESPRIT [8] and digital interferometer direction finding [9] to obtain target's direction before location. If there is a station received weak signal, or interferometer fuzzy cannot correctly solution [10], we may obtain larger error or wrong parameters. The performance of the MLE location method will sharp decline in the presence of measurement error or wrong parameters, because it doesn't have the ability to eliminate error or error parameters.

In addition, MLE location method is not applicable to multiple targets location when target's number is unknown and measured parameters are undistinguished by target. In other words, we can get right location result using MLE location method when measured parameters are only for one target, but we will get wrong location result when measured parameters of multiple targets are undistinguished. If the undistinguished parameters came from more than one target, MLE location method will use all of measured parameters to estimate only one target's position.

In this paper, the position information field for target (PIFT) location method is proposed mainly for multi-target localization. This method maintain good location performance in the presence of larger error or wrong parameters, and suitable for the multi-target localization and determines target's number and position simultaneously even the measured parameters of multi-target are indistinguishable. 


\section{MLE location method}

Assume that the observation platform and radiation sources are located at the ground for convenience of analysis. The passive location system contains $M$ observation platforms which located at $\boldsymbol{q}_{m}=\left(x_{m}, y_{m}\right), m=1,2, \mathrm{~L}, M$, respectively, and radiation source located at $\boldsymbol{X}_{E}=(x, y)$. Measurement parameters of radiation source are $z_{k}, k=1,2, \mathrm{~L}, K$,

$$
z_{k}=h_{k}\left(\boldsymbol{X}_{E}\right)+v_{k} \text {. }
$$

Where $h_{k}\left(\boldsymbol{X}_{E}\right)$ is the theoretical value of measuring parameters $z_{k}$ obtained by station position and target position, and $z_{k}=h_{k}\left(\boldsymbol{X}_{E}\right)$ in an ideal situation. $v_{k}$ is the measurement error of measurement $z_{k}$ which generally have a different distribution depending on the measured value. Take the direction-finding method as an example, the theoretical direction of the first observation platform to target is

$$
h\left(\boldsymbol{X}_{E}\right)=\operatorname{atan}\left(\frac{y-y_{1}}{x-x_{1}}\right) .
$$

$h\left(\boldsymbol{X}_{E}\right)$ is the theoretical value of the azimuth which jointly determined by the position of the first observation platform $\left(x_{1}, y_{1}\right)$ and the position of the target $(x, y)$. However, $z_{k}$ may be a certain type of measurement parameter in the practice positioning process, such as direction of arrival (DOA), time of arrival (TOA), time difference of arrival (TDOA) and so on. $z_{k}$ also can be a composite of various types of parameters. The measured parameters written in matrix form is

$$
\boldsymbol{Z}=\boldsymbol{h}\left(\boldsymbol{X}_{E}\right)+\boldsymbol{v} .
$$

Among them, $\boldsymbol{Z}=\left[\begin{array}{llll}z_{1} & z_{2} & \mathrm{~L} & z_{K}\end{array}\right]^{\mathrm{T}}, \boldsymbol{h}\left(\boldsymbol{X}_{E}\right)=\left[\begin{array}{lllll}h_{1}\left(\boldsymbol{X}_{E}\right) & h_{2}\left(\boldsymbol{X}_{E}\right) & \mathrm{L} & h_{K}\left(\boldsymbol{X}_{E}\right)\end{array}\right]^{\mathrm{T}}, \boldsymbol{v}=\left[\begin{array}{llll}v_{1} & v_{2} & \mathrm{~L} & v_{K}\end{array}\right]^{\mathrm{T}}$.

Assuming no prior knowledge about the target position $\boldsymbol{X}_{E}$, according to the maximum likelihood estimation criteria, the best estimate for $\boldsymbol{X}_{E}$ is achieved when likelihood function of the observation sample $p(\boldsymbol{Z} / \boldsymbol{X})$ to obtain the maximum. Namely,

$$
\hat{\boldsymbol{X}}_{E}=\max _{\boldsymbol{X} \in D_{X}}\{p(\boldsymbol{Z} / \boldsymbol{X})\} .
$$

Where, $p(\bullet)$ represents a probability density function. $\hat{\boldsymbol{X}}_{E}$, the maximum likelihood estimate of $\boldsymbol{X}_{E}$, is the most likely location of $\boldsymbol{X}_{E}$ in the region $D_{X}$ with Observational data $\boldsymbol{Z}$.

If we assume the measurement noise is Gaussian noise with zero mean, then formula (4) can be expressed as

$$
\hat{\boldsymbol{X}}_{E}=\max _{\boldsymbol{X} \in D_{X}}\left\{\frac{1}{(2 \pi)^{K / 2}|\boldsymbol{R}|^{1 / 2}} \exp \left\{-\frac{1}{2}[\boldsymbol{Z}-\boldsymbol{h}(\boldsymbol{X})]^{\mathrm{T}} \boldsymbol{R}^{-1}[\boldsymbol{Z}-\boldsymbol{h}(\boldsymbol{X})]\right\} .\right.
$$

Where, $\boldsymbol{R}=E\left\{\boldsymbol{v} \boldsymbol{v}^{T}\right\}$.Calculate logarithmic of the right formula(5), formula(5)can be further equivalent to

$$
\hat{\boldsymbol{X}}_{E}=\min _{\boldsymbol{X} \in D_{X}}\left\{[\boldsymbol{Z}-\boldsymbol{h}(\boldsymbol{X})]^{\mathrm{T}} \boldsymbol{R}^{-1}[\boldsymbol{Z}-\boldsymbol{h}(\boldsymbol{X})]\right\} .
$$

If we hypothesize that $z_{k}, k=1,2, \mathrm{~L}, K$ are independent of each other, the measurement errors of each parameters are also independent. $\boldsymbol{R}$ can be written as

$$
\boldsymbol{R}=\left[\begin{array}{cccc}
R_{1} & 0 & 0 & 0 \\
0 & R_{2} & 0 & 0 \\
0 & 0 & 0 & 0 \\
0 & 0 & 0 & R_{K}
\end{array}\right] .
$$

Formula (6) can be further written as 


$$
\begin{aligned}
& \hat{\boldsymbol{X}}_{E}=\min _{\boldsymbol{X} \in D_{X}} \sum_{k=1}^{K} d^{2}\left(z_{k}, h_{k}(\boldsymbol{X})\right) \\
& d\left(z_{k}, h_{k}(\boldsymbol{X})\right)=\left\{\left[z_{k}-h_{k}(\boldsymbol{X})\right]^{\mathrm{H}} R_{k}^{-1}\left[z_{k}-h_{k}(\boldsymbol{X})\right]\right\}^{\frac{1}{2}} .
\end{aligned}
$$

\section{PIFT location method}

From equation (6) we can see that all of the observed parameters are used to estimate the location of the target, or if some observed parameters with larger error or wrong, it may lead to a larger target location error, even the positioning error. Therefore, we introduce a cost function to filter parameter information. Equation (8) can be modified to

$$
\begin{aligned}
& \hat{\boldsymbol{X}}_{E}=\min _{\boldsymbol{X} \in D_{X}} \sum_{k=1}^{K} C\left[d^{2}\left(z_{k}, h_{k}(\boldsymbol{X})\right)\right] \\
& d\left(z_{k}, h_{k}(\boldsymbol{X})\right)=\left\{\left[z_{k}-h_{k}(\boldsymbol{X})\right]^{\mathrm{H}} R_{k}^{-1}\left[z_{k}-h_{k}(\boldsymbol{X})\right]\right\}^{\frac{1}{2}}
\end{aligned}
$$

Where, $C[\bullet]$ is a cost function. The role of the cost function is to filter parameter information, remove erroneous data, and reduce the impact of large errors data on the positioning results estimation. If the measurement noise is Gaussian distribution, measurement data can be denoted as $z_{k} \sim N\left(\mu_{k}, R_{k}\right)$. Since the data standard error of Gaussian process is mainly less than $3 \sqrt{R_{k}}$. We can make $\gamma=3 \sqrt{R_{k}}$, then the cost function can be selected as follows

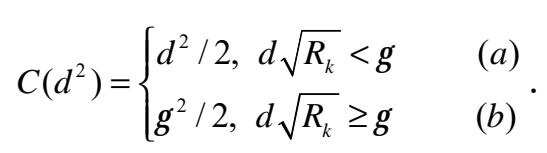

The cost function can be defined as limited squared cost function. Cost function can have a variety, but positioning results of different cost function is slightly. It can be used as a cost function as long as it has the function of screening data in theoretically. A variety of cost function and even can be used in cascade. Without loss of generality, measuring a large number of same type parameters relating to target can be considered as Gaussian distribution during positioning. Therefore, we use limited squared cost function in the later discussion.

Equation (9) and (10) can also be written as

$$
\begin{aligned}
& \hat{\boldsymbol{X}}_{E}=\min _{\boldsymbol{X} \in D_{X}} \sum_{k=1}^{K} C\left[d\left(z_{k}, h_{k}(\boldsymbol{X})\right)\right] \\
& d\left(z_{k}, h_{k}(\boldsymbol{X})\right)=\left\{\left[z_{k}-h_{k}(\boldsymbol{X})\right]^{H} R_{k}^{-1}\left[z_{k}-h_{k}(\boldsymbol{X})\right]\right\}^{\frac{1}{2}}, \\
& C(d)=\left\{\begin{array}{ll}
d^{2} / 2, d \sqrt{R_{k}}<\gamma & (a) \\
\gamma^{2} / 2, & d \sqrt{R_{k}} \geq \gamma
\end{array} .\right.
\end{aligned}
$$

Assuming that the prior probability density of target located at $\boldsymbol{X}$ is $p_{0}(\boldsymbol{X})$, the PIFT can be defined as

$$
f_{X}(\boldsymbol{X} / \boldsymbol{Z})=\max _{\mathbf{X} \in D_{X}}\left\{\ln p_{0}(\boldsymbol{X})-\sum_{m=1}^{M} C\left[d\left(z_{m}, h_{m}(\boldsymbol{X})\right)\right]\right\} .
$$

From equation (12) we can see that cost function will satisfies the equation (12)-(a) in the vicinity of the target point if the parameter measurement error is small. Conversely, if parameter measurement error is large or wrong, cost function will satisfies the equation (12)-(a) in the vicinity of the target point. Thus, cost function will lose its function in the ideal case of a single target location with all the measured parameters are very high accuracy. The PIFT location method will equivalent to the MLE location method in this case and has a good positioning performance. 
However, if part of the parameter measurement error is large or wrong, MLE location method will use all of the observed parameters to estimate the positioning of the target and get poor or wrong positioning results. The PIFT location method will better positioning results in this case by using the cost function to filter parameter information, remove erroneous data, and reduce the impact of large errors data on the positioning results estimation. The PIFT location method is no longer equivalent to the MLE location method in this case.

In addition, The MLE location method is not applicable to multiple targets location when target's number is unknown and measured parameters are undistinguished by target even with high accuracy measured parameters. The MLE location method will not get the location of multiple targets because of the number of target location is unknown and the measured parameters are indistinguishable, it just gets a most consistent target location with bulk of the measurement parameters. The PIFT location method can achieve indistinguishable multiple targets location with a cost function, unlike the MLE location method.

\section{PIFT location method implementation steps}

The positioning process of the PIFT location method can be divided into two steps. First, select location region and divide into grids. Second, determine the number and location of the target.

Select location area and divide into grids. Before using PITFT location method, we should select an area of interest may exist locate targets according to some priori information. The area shape can be square, circular or fan-shaped and so on. Taking the square area as an example, choosing Cartesian coordinate system, and assuming that an edge of the square area parallel to the $\mathrm{X}$ axis of the coordinate system. The area range can be expressed as:

$$
D_{X}=\left[x_{\min }, x_{\max }\right] \otimes\left[y_{\min }, y_{\max }\right] .
$$

Dividing the area into a uniform grid $N_{x} \times N_{y}$ in steps of $\Delta x$ and $\Delta y$ in the $\mathrm{X}$-axis and $\mathrm{Y}$-axis by positioning accuracy, $N_{x}$ and $N_{y}$ can be calculated as

$$
N_{x}=\left\lceil\frac{x_{\max }-x_{\min }}{\Delta x}\right\rceil, N_{\mathrm{y}}=\left\lceil\frac{y_{\max }-y_{\min }}{\Delta y}\right\rceil .
$$

Where, $\lceil\bullet\rceil$ is rounded up. Calculated for each grid point coordinates $\left(x_{n_{x}}, y_{n_{y}}\right)$,

$$
\begin{aligned}
& x_{n_{x}}=x_{\text {min }}+n_{x} \Delta x, \quad y_{n_{y}}=y_{\text {min }}+n_{y} \Delta y \\
& n_{x}=0,1, \mathrm{~L}, N_{x}, n_{y}=0,1, \mathrm{~L}, N_{y}
\end{aligned}
$$

Determine the number and location of the target. Calculate measurement parameters theoretical values $h_{k}(\boldsymbol{X})$ of observatory for each grid point $\boldsymbol{X}=\left(x_{n_{x}}, y_{n_{y}}\right)$ firstly. Then PIFT $f_{X}$ is calculated by formula (13) for each grid point based on the measured parameters and the cost function. Search number of peaks of PIFT $f_{X}$ in the area $D_{X}$. The position of maximum peak $\left(n_{x}, n_{y}\right)=\left[n_{x}(1), n_{y}(1)\right]$ can be expressed as:

$$
\left[n_{x}(1), n_{y}(1)\right]=\underset{n_{x}, n_{y}}{\arg \max }\left\{f_{X}\left(n_{x}, n_{y}\right)\right\} .
$$

The maximum peak is

$$
f_{\text {peak }}(1)=\left.f_{X}\left(n_{x}, n_{y}\right)\right|_{\left(n_{x}, n_{y}\right)=\left[n_{x}(1), n_{y}(1)\right]}
$$

Sorting the other peak position by the peak size, and reserving the largest $P$ if the following conditions are met,

$$
f_{\text {peak }}(p)=\left.f\left(n_{x}, n_{y}\right)\right|_{\left(n_{x}, n_{y}\right)=\left[n_{x}(p), n_{y}(p)\right]}>\gamma_{p} \cdot f_{\text {peak }}(1) .
$$


Where, $p=1,2, \mathrm{~L}, P, \quad \gamma_{p}$ is a threshold, $0<\gamma_{p}<1$. Now determine the number of target needed to locate is $P$. The sequence number of grid point corresponds to each target is

$$
\left[n_{x}(1), n_{y}(1)\right],\left[n_{x}(2), n_{y}(2)\right], \mathrm{L},\left[n_{x}(P), n_{y}(P)\right] \text {. }
$$

The initial position of the target $p$ may be initially estimated as

$$
x_{p, 0}=x_{\min }+n_{x}(p) \Delta x, \quad y_{p, 0}=y_{\min }+n_{y}(p) \Delta y .
$$

Measurement parameters can be classified according to the target on $P$ goals for the same treatment. Then we can get each target's position and orientation error by iterative method using initial point obtained by PIFT location method.

\section{Experiments}

The positioning performances of the PIFT location method and MLE location method are analyzed by multi-station direction-finding method. Assume five stations, numbered $1,2,3,4,5$, located at $(0,0) \mathrm{km}$, $(25,0) \mathrm{km},(50,0) \mathrm{km},(75,0) \mathrm{km}$ and $(100,0) \mathrm{km}$, respectively.

Experiment 1: Single target situation. Assuming an area with only one target, which position is $(x, y)=(35,60) \mathrm{km}$. The target's positioning is obtained by the PIFT location method and MLE location method respectively using direction information. Monte-Carlo test method is used to measure the positioning performance, the mean square error of $N$ times Monte-Carlo experiment is

$$
\operatorname{RMSE}=\sqrt{\frac{1}{N} \sum_{n=1}^{N}\left[\left(\hat{x}_{n}-x\right)^{2}+\left(\hat{y}_{n}-y\right)^{2}\right]} .
$$

Among them, $\left(\hat{x}_{n}, \hat{y}_{n}\right)$ is the nth estimation position of the target. Assuming observatory $1 \sim 4$ can get more accurate direction of arrival (DOA), which direction finding precision fixed to one degree. The direction finding precision of station 5 changes from one to ten degrees. The purpose of this experiment is to study the positioning performance of two location method in the presence of big measurement error or even wrong observation data.

In the process of positioning calculation using PIFT location method, we think $R_{1}=R_{2}=\mathrm{L}=R_{K}=R$ are equal, and all equal to one degree, in other words, the precision of direction finding is hold to one degree. The reason why we did this assumption is that we don't know great measurement error or wrong data are contained in observation data in advance, which is consistent with actual direction positioning process. Fig. 1 shows the relations between RMSE of two location methods and the accuracy of direction finding of 5th station with 500 Monte-Carlo experiments.

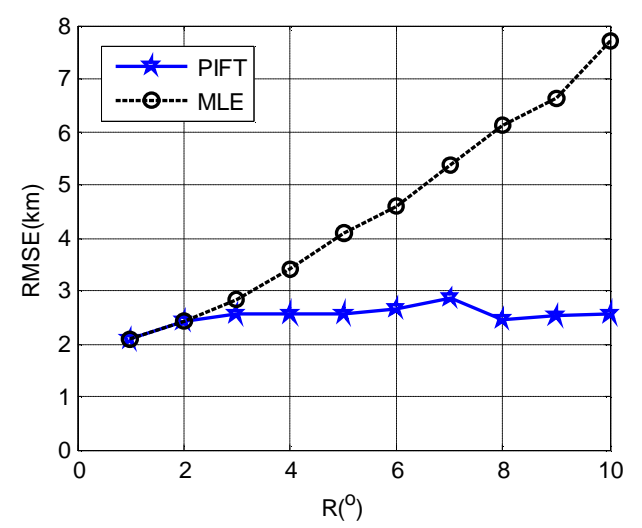

Fig.1 Relation between RMSE and the accuracy of direction finding with a single target

The two location methods can maintain better positioning accuracy in fig. 1 when each observatory direction finding precision is higher. However, the positioning performance of MLE location method drops rapidly with the deterioration of an observatory direction finding precision, and PIFT location method still able to maintain good positioning performance. 
Experiment 2: multiple target situations. Assuming that there is two targets exist in an area, the first target's position is $\left(x_{1}, y_{1}\right)=(35,60) \mathrm{km}$, and the second target's position is $\left(x_{2}, y_{2}\right)=(65,60) \mathrm{km}$. Ten directions of arrival data are obtained by five stations with direction finding error of one degree. But we can't able to determine which five directions of arrival is about first target or second target, in other words, measured data cannot be classified according to the target. The positioning results of these data using MLE location method and PIFT location method are shown in figure 2 and figure 3, respectively.

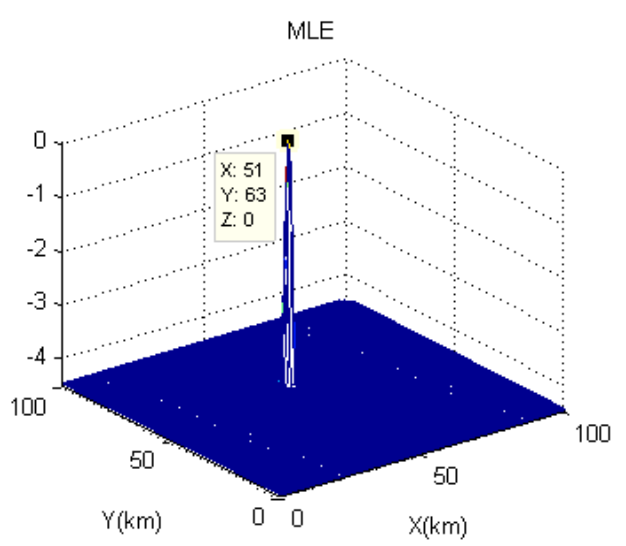

Fig.2 MLE location method

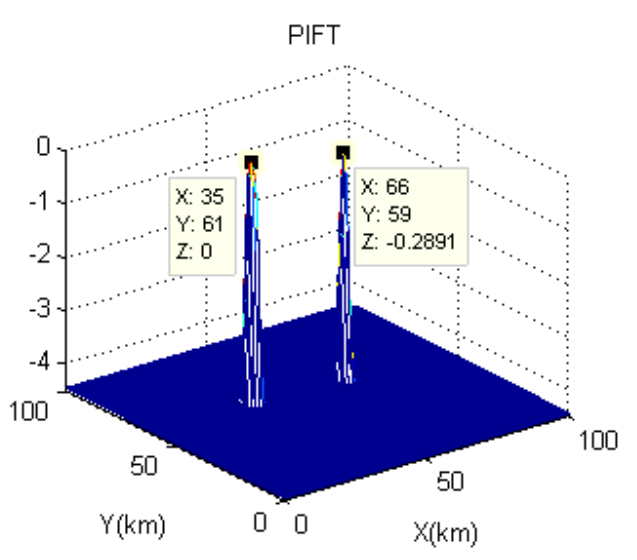

Fig.3 PIFT location method

Comparison of fig. 2 and fig. 3 we can find that the MLE location method can only get one target's position, and the estimated target's position is wrong, which is approximately the middle of the true position of the two targets. This indicates that the MLE location method does not have the ability for multiple targets location. The PIFT location method can determine the target's number is two, the position of the two targets are close to the real position respectively within the allowed error range, which indicate that the PIFT location method has the ability to multiple targets location when measurement parameters used to locate are indistinguishable.

Reducing the distance between the two targets, the second target's position change to $(45,60) \mathrm{km}$. Suppose station 4 and 5 can only obtain one direction of arrival about the first target and second target, respectively, other observatories are measured two orientations, direction finding error is also one degree. In practical application, the number of targets usually unknown, the orientations measured by stations 4 and 5 cannot determine whether belonging to the same goal. The positioning results of two location method in the case of information lost are shown in fig. 4 and fig.5.

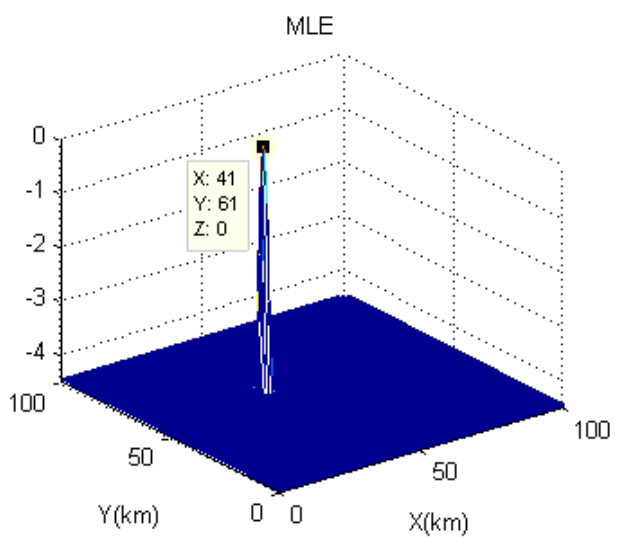

Fig.4 MLE location method with information lost

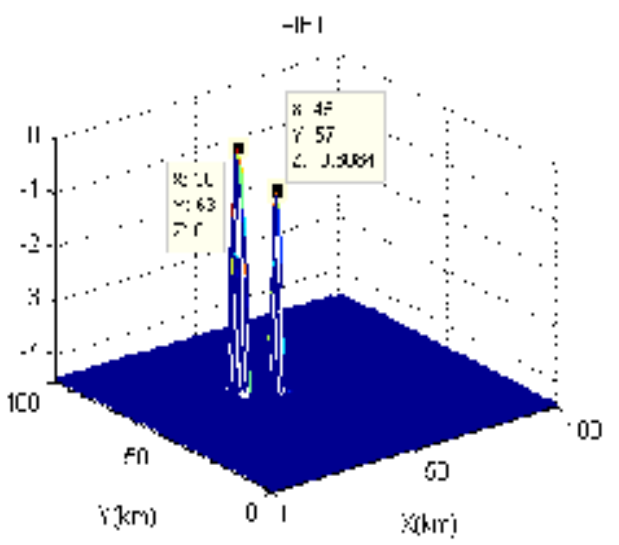

Fig.5 PIFT location method with information lost

Through the figures 4 and 5 can be found that when the two targets are relatively close, the MLE location method can only estimate the position of one target, the wrong location results are obtained while the PIFT location method can still distinguish the number of targets and realize positioning. But when multiple targets are too close, PIFT location method also may be unable to distinguish between the target and the multiple targets may be sentence to one target. 


\section{Conclusions}

The positioning performance of MLE location method fell sharply in the process of single target location when some parameters measurement error or wrong, and it cannot realize positioning when parameters about multiple targets are indistinguishable. The PIFT location method has been raised in this paper. It has stable performance and strong robustness by adding cost function, which can reduce the impact of large error or wrong data to positioning results. The PIFT location method can obtain the number and location of targets at the same time in fixing the indistinguishable multiple targets. The indistinguishable parameters can be classified according to the target number and location in the subsequent processing, and the indistinguishable multiple targets positioning problem is transformed into single target positioning problem.

\section{References}

[1] Lee J Y, Hudson R E, Yao K. Acoustic DOA Estimation: An Approximate Maximum Likelihood Approach [J]. Systems Journal, IEEE , 2014, 8(1): 131-141.

[2] Shen J Y, Molisch A, Salmi J. Accurate passive location estimation using TOA measurements [J]. IEEE Transactions on Wireless Communications. 2012, 11(6): 2182-2192.

[3] Yuan G, Chen J. Solution on ambiguity of time difference location system of three stations [J]. Journal of CAEIT, 2014, 9(1): 89-92.

[4] Picard J S, Weiss A J. Time difference localization in the presence of outliers [J]. Signal Process, 2012, 92(10): 2432-2443.

[5] Wang Z, Luo J A, Zhang X P. A novel location-penalized maximum likelihood estimator for bearing-only target localization [J]. IEEE Transactions on Signal Processing, 2012, 60(12): 6166-6181.

[6] Bai J, Wang G H, Wang N, et al. Study on optimum cut angles in bearing-only location systems [J]. Acta Aeronautica Et Astronautica Sinica, 2009, 30(2): 298-304.

[7] Cai J J, Bao D, Li P, et al. Two-dimensional DOA estimation using reduced-dimensional MUSIC algorithm with strong-constraint optimization[J]. Journal of Electronics \& Information Technology, 2014, 36(5): 1113-1118.

[8] Chen C, Zhang X F. A RD-ESPRIT algorithm for coherent DOA estimation in monostatic MIMO radar using a single pulse [J]. International Journal of Electronics, 2014, 101(8): 1074-1085.

[9] Zhang M, Guo F C, Zhou Y Y, et al. Direction finding method for two-dimension interferometer using the time varying long baseline [J]. International Journal of Electronics, 2013, 35(12): 2882-2888.

[10] Han Y T, Wu S L, Ma L, et al. Ambiguity-resolving method of interferometer based on phase difference with vector average [J]. Journal of Electronic Measurement and Instrument, 2011, 25(10): 842-849.

[11] Ma X T, Luo J Q, Zhang K. A location method for multi-target by position information field based on DOA [J]. Journal of Signal Processing, 2013, 29(1): 121-126. 